\title{
Investimentos em Tecnologia da Informação e Impactos na Produtividade Empresarial: uma Análise Empírica à Luz do Paradoxo da Produtividade
}

\section{Information Technology Investments and Impact on the Productivity of Firms: an Empirical Analysis in Light of the Productivity Paradox}

Ivan Ricardo Gartner * Doutor em Engenharia de Produção pela UFSC. Professor do PPGA/UMESP, São Bernardo do Campo/SP, Brasil.

Ronaldo Zwicker Doutor em Administração pela FEA/USP. Professor da FEA/USP, São Paulo/SP, Brasil.

Wilhelm Rödder

Doutor em Ciências Econômicas pela RWTH Aachen, Alemanha. Professor-Catedrático na Faculdade de Ciências Econômicas da FernUniversität in Hagen, Alemanha.

* Endereço: Ivan Ricardo Gartner

Universidade Metodista de São Paulo, Programa de Pós-Graduação em Administração, Rua Alfeu Tavares, 149, Bloco Capa, Rudge Ramos, São Bernardo do Campo/SP, 09641-000. E-mail: ivan.gartner@metodista.br

Copyright (C 2009 RAC. Todos os direitos, inclusive de tradução, são reservados. É permitido citar parte de artigos sem autorização prévia desde que seja identificada a fonte. 


\title{
RESUMO
}

Este trabalho objetivou testar a ocorrência do Paradoxo da Produtividade em uma amostra de empresas brasileiras; este Paradoxo estabelece que os acréscimos nos investimentos em tecnologia da informação não são acompanhados por acréscimos na produtividade das empresas. A partir de uma amostra de 429 observações de 98 empresas brasileiras que, no período de 2000 a 2006, faturaram cerca de US\$ 856 bilhões e operaram investimentos em tecnologia da informação de US\$ 12,9 bilhões, foi testado um modelo baseado na função de produção de Cobb-Douglas, que apresentou indícios de que o acréscimo neste tipo de investimento foi acompanhado de um acréscimo positivo nas receitas. $\mathrm{O}$ trabalho mostrou, ainda, indícios de que houve eficiência marginal nos investimentos em tecnologia da informação e de que a relação entre as receitas e o número de funcionários empregados em TI alcançou um aparente ponto de saturação. $\mathrm{Na}$ análise dos resultados foram identificados comportamentos distintos entre os setores analisados e nas conclusões foram apresentados exemplos de como os resultados poderiam ser aplicados no suporte ao processo decisório do planejamento de investimentos e de estratégias de negócios em tecnologia da informação.

Palavras-chave: investimentos em tecnologia da informação; retorno sobre os investimentos; Paradoxo da Produtividade; função Cobb-Douglas; dados em painel não-balanceado.

\begin{abstract}
This study aimed to investigate the occurrence of the Productivity Paradox of information technology investments in a sample of Brazilian firms. This Paradox establishes that the increase in information technology investments is not accompanied by the increase of firms' productivity. The estimated model was based on the Cobb-Douglas production function and considered a sample of 429 observations of 98 Brazilian firms that had aggregated sales of over US\$ 856 billion and had operated information technology investments around of US\$ 12.9 billion during the period of 2000 to 2006 . The estimated model showed evidence that changes in the information technology investments were statistically correlated with changes in productivity gains. In addition, the model showed that there was marginal efficiency in the information technology investments and that the functional relationship between production and the number of employees in information technology reached an apparent saturation point. The empirical results showed different behaviors among analyzed sectors and the findings were presented with examples of how the results could be applied to support information technology investments and business strategy processes.
\end{abstract}

Key words: information technology investments; return on investment; Productivity Paradox; Cobb-Douglas function; unbalanced panel data. 


\section{INTRODUÇÃO}

A importância da tecnologia da informação [TI] pode ser claramente identificada por seu efeito direto na atividade econômica, que pode ser representado pelo crescimento constante da participação das empresas de TI na produção nacional. Embora a análise desse efeito direto da TI seja importante, há relevância na análise de seus efeitos indiretos na economia, ao se enfocar seus impactos no desempenho das organizações que a absorvem.

Ao se estabelecer que as empresas absorvem a TI por meio dos investimentos em tecnologia da informação [ITI], pode-se situar essa análise de efeitos indiretos no âmbito da análise microeconômica. Nesse caso, o foco da análise econômica situa-se na mensuração do retorno dos ITI, operados por cada empresa ou grupo de empresas, sendo esses investimentos aqui definidos como gastos em hardware e seus aplicativos, software e seus recursos, sistemas de telecomunicações, gestão de dados e informações que possibilitam às empresas aumentar a velocidade da geração, difusão e uso dos novos conhecimentos necessários a sua integração às constantes mutações no mercado. Essa integração refere-se à aquisição da informação necessária ao gerenciamento das mudanças, adaptações e atualizações da tecnologia de produção e de serviços, bem como à gestão de sua participação na cadeia econômica, pela otimização da aquisição de seus insumos e da distribuição de seus produtos e serviços.

Sob essa ótica, o relacionamento do alcance dos objetivos globais das estratégias competitivas empresariais com o volume de investimentos em tecnologia da informação parece guardar uma relação lógica de causa e efeito. No entanto, é necessário analisar com maior profundidade essa questão e procurar identificar a existência de um ponto de saturação, a partir do qual os acréscimos nos ITI apresentam um decréscimo em seus retornos.

Devido às suas especificidades e ao seu valor estratégico, a análise do retorno dos ITI demanda o desenvolvimento de novas ferramentas de avaliação, que devem ser condicionadas pelas características do setor de atuação das organizações estudadas, e cumpre considerar questões como a intensidade do uso de mão-de-obra qualificada e os investimentos em pesquisa e desenvolvimento $[\mathrm{P} \& \mathrm{D}]$.

Estudos empíricos de abrangência setorial sobre o retorno dos ITI têm sido realizados em países desenvolvidos, especialmente nos Estados Unidos e em países europeus, e têm apresentado resultados conflitantes sobre a existência de significância estatística entre o aumento da produtividade das empresas e o aumento dos ITI. Essas evidências têm reforçado a existência de um possível Paradoxo de Produtividade, segundo o qual o aumento no volume dos investimentos em tecnologia da informação não é acompanhado pelo aumento na produtividade das empresas.

O Paradoxo da Produtividade foi estabelecido a partir das conclusões de estudos macroeconômicos realizados por Robert Solow, que culminaram com sua Teoria do Crescimento (Solow, 1988). Tal estudioso ficou conhecido na área de TI por sua frase "you can see the computer age everywhere but in the productivity statistics" (Solow, 1987, p. 36), que instigou vários pesquisadores a tratar da avaliação do retorno dos investimentos em tecnologia da informação, porquanto seu comentário apresentou um questionamento quanto à viabilidade dessa modalidade de investimento. $\mathrm{O}$ debate em torno dessa temática teve seu apogeu na segunda metade dos anos 90, mas ainda faz parte da agenda de pesquisadores de áreas como economia da informação, administração de sistemas e de tecnologia da informação.

Embora a proposta de Solow tenha caráter macroeconômico, os estudos têm sido desenvolvidos também do ponto de vista microeconômico. Uma pesquisa feita nos artigos publicados nos anais dos encontros científicos da ANPAD e em periódicos de alta classificação como RAC, RAUSP, RAE, Contabilidade e Finanças, mostrou que são poucas as pesquisas desenvolvidas no Brasil sobre o assunto, com destaque para algumas iniciativas no âmbito microeconômico, como o trabalho de Menezes e Moura (2004), que tratou de um estudo de caso de um banco brasileiro, e de Teixeira 
(1998), que apresentou uma revisão bibliográfica do ponto de vista macroeconômico, com ênfase na explicação do Paradoxo, a partir dos processos de difusão e aprendizagem das inovações tecnológicas e organizacionais.

A carência de estudos empíricos desenvolvidos para a validação do paradoxo da produtividade no Brasil motivou a elaboração deste trabalho, que objetivou investigar, em âmbito microeconômico, a correlação entre os investimentos em tecnologia da informação [ITI] e a produtividade empresarial. A principal hipótese deste trabalho tratou de identificar e analisar a ocorrência do Paradoxo da Produtividade em uma amostra de empresas brasileiras, considerando que tal Paradoxo estabelece que (H1) os investimentos em tecnologia da informação têm um impacto negativo ou nulo na produtividade empresarial (Pilat, 2004), ceteris paribus. Como complemento a essa suposição geral, foram estabelecidas outras duas hipóteses, mais específicas, que igualmente divergem da lógica econômica dos estudos de viabilidade econômica de investimentos, e que vão ao encontro dos pressupostos do Paradoxo, que são: (H2) os rendimentos de escala do conjunto de insumos de tecnologia da informação são decrescentes e (H3) não há eficiência marginal do capital investido em tecnologia da informação.

O trabalho está estruturado em cinco partes. A primeira parte refere-se a essa introdução, onde consta a motivação, a problemática, o sistema de hipóteses e o objetivo do trabalho. Na segunda parte é apresentada a síntese de uma revisão bibliográfica dos estudos empíricos realizados sobre o assunto. $\mathrm{Na}$ terceira parte tem-se a sustentação teórica do estudo empírico proposto. Na quarta parte tem-se a descrição dos procedimentos metodológicos adotados, a análise empírica e a análise da eficiência econômica dos investimentos em tecnologia da informação. Por fim, são apresentadas as considerações finais do estudo.

\section{SÍNTESE DOS ESTUDOS EMPÍRICOS SOBRE O RETORNO dOS INVESTIMENTOS EM TECNOLOGIA DA INFORMAÇÃO}

O aumento dos investimentos em tecnologia da informação [ITI] foi uma resposta ao esgotamento do modelo de produção que vigorou até o final da Segunda Guerra Mundial. Os vários sinais de exaustão identificados na época, como desaceleração do crescimento da produtividade, rejeição dos trabalhadores ao autoritarismo patronal, rigidez das linhas de produção, incapacidade de reduzir os custos de produção, encarecimento das matérias-primas e das fontes energéticas, foram agravados quando o mercado passou a ser regido pela demanda e não mais pela oferta.

A partir de então, os processos técnico-científicos passaram a ter maior aplicação no aumento da produtividade industrial e teve início o processo de integração dos sistemas produtivos mediante inovações revolucionárias e difusão de novas tecnologias. As empresas passaram a fazer acordos de cooperação científico-tecnológica, o que propiciou o acirramento do processo competitivo, ocasionando uma nova reconfiguração da economia mundial, baseada na incorporação de novas tecnologias, cujas escalas de produção são cada vez mais globalizadas (Santos, 1987). Conseqüentemente, a tecnologia e a informação passam a formar a mola propulsora da economia mundial e seu domínio pode determinar a sobrevivência de organizações e instituições em geral.

Banker e Kauffmann (2004) realizaram uma revisão bibliográfica extensa com o objetivo de analisar a produção científica no campo da administração, que abordou o incremento dos ITI ocorridos no PósGuerra e seus desdobramentos na gestão das organizações. Seu trabalho culminou com a divisão das pesquisas realizadas em cinco grupos: (a) Ciência e apoio à decisão, que agrega os estudos referentes à aplicação de computadores no apoio, controle e processos de tomada de decisão gerencial; (b) Valor da informação, que abrange os estudos que tomam por base a análise econômica da informação como sendo uma commodity na administração de uma empresa; (c) Desenho de sistemas homem-máquina, que enfatizam a base cognitiva para o desenho de sistemas eficientes; (d) Estratégia e organização de sistemas de informação, que enfoca o nível de análise local do valor do investimento em sistemas de 
informação, ao invés das percepções do sistema ou de seu usuário; (e) Economia da tecnologia e dos sistemas de informação, que trata da aplicação de conceitos teóricos e métodos da economia analítica e empírica aos problemas gerenciais que envolvem tecnologia e sistemas de informação.

No âmbito do grupo de pesquisas sobre economia da tecnologia e dos sistemas de informação (e) tem-se a aplicação do ferramental da economia para a mensuração e análise dos impactos da tecnologia da informação nas organizações sob o enfoque da eficiência técnica e econômica. É nesse grupo de estudos que se incluem os artigos publicados por Brynjolfsson e Hitt (1995, 1996, 1998, 2000), Brynjolfsson e Yang (1996), Brynjolfsson (1993), todos causadores de grande repercussão no meio acadêmico, uma vez que seus resultados refutaram o Paradoxo da Produtividade de Solow. Os pesquisadores realizaram vários estudos para avaliar a correlação entre os investimentos em tecnologia da informação e os ganhos de produtividade de várias amostras de grandes empresas americanas de vários setores industriais. Nesse estudo, os gastos em TI apresentaram uma substancial e estatisticamente significante contribuição no desempenho das empresas analisadas, o que estimulou outros pesquisadores a desenvolver pesquisas semelhantes, muitas delas também refutando o Paradoxo da Produtividade.

O estudo realizado por Bharadwaj, Bharadwaj e Konsynski (1999) concluiu que os ITI têm uma relação estatisticamente significante com uma medida de produtividade empresarial baseada no mercado, utilizando o índice $q$ de Tobin. Esse estudo teve como amostra um grande número de indústrias no período de 1988 e 1993 e concluiu que, em todos os anos analisados, os ITI tiveram uma associação positivamente significante com o valor $q$ de Tobin.

Milana e Zeli (2002) examinaram o impacto da tecnologia da informação na eficiência técnica produtiva de grande número de indústrias italianas, que foram distribuídas em quatro grupos, de acordo com a intensidade de gastos em pesquisa e desenvolvimento [P\&D]. Foi realizado um estudo correlacional entre variáveis descritoras de investimentos em hardware e software e um índice de eficiência técnica produtiva empresarial; esse índice foi mensurado por meio da análise envoltória de dados [DEA]. Os autores concluíram que essa correlação não é significativamente rejeitada na maioria dos setores industriais analisados e que, em geral, correlações positivas não são rejeitadas em todos os quatro grupos de indústrias. No entanto, a eficiência técnica não parece ter sido afetada em um significativo grupo de indústrias caracterizadas por alto dispêndio em $\mathrm{P} \& \mathrm{D}$, o que pode significar que as indústrias de alto grau de inovação têm pequena margem de benefícios a extrair de seus investimentos em tecnologia da informação [ITI].

Uma forma alternativa de se testar o Paradoxo da Produtividade foi feita pelos pesquisadores Engelbrecht e Xayavong (2006), que agregaram a TI e os gastos com sistemas de comunicação, resultando no termo tecnologia da informação e comunicação [TIC]. O estudo buscou correlacionar o impacto da TIC no crescimento da produtividade do trabalho de uma amostra de 29 indústrias da Nova Zelândia, no período de 1988 a 2003. Os autores desenvolveram uma metodologia para classificar as indústrias da amostra quanto à maior ou menor intensidade de TIC e analisaram a produtividade do trabalho nesses dois grupos. Os resultados comprovaram que as indústrias com uso mais intensivo de TIC apresentaram um maior crescimento da produtividade do trabalho do que aquelas de uso menos intensivo.

Estudo nessa mesma linha de abordagem foi conduzido por Atzeni e Carboni (2006), que apresentou um estudo econométrico sobre a relação entre a TIC em um grande número de empresas industriais italianas no período de 1995 a 1997. Por meio de uma modelagem econométrica robusta, o estudo mostrou que as indústrias mais inovadoras, em termos tecnológicos, apresentaram maior produtividade da tecnologia da informação e comunicação. Além disso, o estudo concluiu que os investimentos em tecnologia da informação e comunicação têm maior impacto no crescimento das indústrias do que os demais investimentos operados. 


\section{FUNDAMENTAÇÃO TEÓRICA dOS ESTUdOS EMPÍRICOS SOBRE O RETORNO DOS INVESTIMENTOS EM TECNOLOGIA DA INFORMAÇÃO}

A fundamentação teórica dos estudos empíricos sobre o retorno dos ITI que visam validar o Paradoxo da Produtividade pode encontrar alicerce no arcabouço microeconômico da teoria da produção, conforme estudos de Brynjolfsson (1993), Brynjolfsson e Hitt (1995, 1996, 1998, 2000), Brynjolfsson e Yang (1996), Gurbaxani, Melville e Kraemer (1998, 2000), Peslak (2003), O’Mahony e Vecchi (2005), e Sabyasachi (2005).

O ponto de partida desta análise é a construção de uma relação funcional da produção que, segundo Humphrey (1997), toma por base a teoria da utilidade, assumindo a forma:

$Y=f(L, K, T, \ldots)$

pela qual o volume de produção $Y$ resulta da combinação dos fatores trabalho $L$, capital $K$, terra $T$ e de outros insumos. No âmbito individual das firmas, essa função de produção expressa uma relação tecnológica, ou progresso técnico, para que os tomadores de decisão encontrem a combinação ótima dos insumos que devem ser usados para produzir um dado nível de produto ao custo mais baixo, dado seu atual estágio de desenvolvimento tecnológico.

A equação genérica (1) pode ser adaptada ao objetivo deste trabalho, que testará a hipótese da ocorrência do Paradoxo de Produtividade, considerando a seguinte relação funcional entre a produção de cada empresa e seus insumos de tecnologia da informação:

$Y_{i}=f\left(L_{i}, K_{i 1}, K_{i 2}, K_{i 3}\right)$,

onde $Y_{i}$ é o valor da produção anual vendida, em unidades monetárias, $L_{i}$ é o número de pessoas empregadas na área de TI, $K_{i l}$ é valor do investimento anual em tecnologia da informação, em unidades monetárias, $K_{i 2}$ é o valor do estoque físico de hardware constituído por microcomputadores e periféricos e $K_{i 3}$ é o valor do estoque físico de hardware constituído por servidores, e todas as variáveis se referem à empresa analisada $i$.

A adequação teórica dessa relação funcional da produção foi proposta originalmente por Cobb e Douglas (1928), pela incorporação da constante $A$ e dos coeficientes de elasticidade às variáveis $L_{i}$, $K_{1 i}, K_{2 i}, K_{3 i}$, o que resulta em:

$Y_{i}=A L_{i}^{\alpha} K_{1 i}^{\beta_{1}} K_{2 i}^{\beta_{2}} K_{3 i}^{\beta_{3}}$,

Essa formulação resulta no grau de homogeneidade ou elasticidade de escala da função de produção. $\mathrm{O}$ parâmetro $A$ indica a eficiência estimada do processo produtivo, pois corresponde à produtividade total dos fatores. Mudanças nesse parâmetro, mantendo-se constantes os demais, levam a uma mudança proporcional uniforme da produção para cada combinação dos fatores, o que pode ser comprovado pelo rearranjo da equação (3), onde $A=Y_{i} / L_{i}^{\alpha} K_{1 i}^{\beta_{1}} K_{2 i}^{\beta_{2}} K_{3 i}^{\beta_{3}}$. De acordo com Chiang (1974), os expoentes da equação medem os retornos de escala, pois esta é uma função homogênea de grau $\alpha+\beta_{1}+\beta_{2}+\beta_{3}$, pois $\left[f\left(\lambda L, \lambda K_{1}, \lambda K_{2}, \lambda K_{3}\right)=\lambda_{\alpha+\beta_{1}+\beta_{2}+\beta_{3}} f\left(L, K_{1}, K_{2}, K_{3}\right)\right]$. Logo, para uma análise de longo prazo, quando todos os fatores são variáveis, tem-se que há retornos crescentes de escala quando $\alpha+\beta_{1}+\beta_{2}+\beta_{3}>1$; há retornos constantes de escala quando $\alpha+\beta_{1}+\beta_{2}+\beta_{3}=1$; e há retornos decrescentes de escala quando $\alpha+\beta_{1}+\beta_{2}+\beta_{3}<1$.

Em termos operacionais, a função Cobb-Douglas é especialmente atrativa para o processo de estimação estatística, porque, de acordo com Baumol (1977), ela tem a propriedade de se tornar linear, 
quando reescrita em termos do ajuste logarítmico de suas variáveis, resultando na seguinte função loglinear:

$\ln Y_{i}=\ln A+\alpha \ln L_{i}+\beta_{1} \ln K_{1 i}+\beta_{2} \ln K_{2 i}+\beta_{3} \ln K_{3 i}$,

A relação funcional de produção e insumos da equação (4) sintetiza os preceitos da teoria econômica neoclássica tradicional, a partir dos quais serão realizados os testes empíricos visando à validação do Paradoxo da Produtividade.

\section{ANÁlISE EMPÍRICA DO RETORNO dOS INVESTIMENTOS EM TECNOLOGIA DA INFORMAÇÃO NO BRASIL}

\section{Especificação do Modelo Econométrico}

A especificação econométrica que procurará representar o modelo teórico equacionado requer que se extraia a primeira diferença das variáveis logaritmizadas de (4), resultando em:

$\Delta \ln Y_{i}=\ln A+\alpha_{1} \Delta \ln L_{i}+\beta_{1} \Delta \ln K_{1 i}+\beta_{2} \Delta \ln K_{2 i}+\beta_{3} \Delta \ln K_{3 i}+\varepsilon_{i}$,

o que permite a estimação dos parâmetros pela análise de regressão múltipla.

Os parâmetros $\alpha_{1}, \beta_{1}, \beta_{2}$ e $\beta_{3}$ podem ser estimados pelo método dos mínimos quadrados ordinários que, em geral, são operacionalizados por análises transversais (cross-section). Nesse tipo de análise, estima-se a relação funcional entre as variáveis em função do conjunto das empresas da amostra. Como os dados disponíveis são publicados com periodicidade anual, o ideal é que se recorra uma especificação que considere a dinâmica da evolução temporal das variáveis estudadas de cada empresa, o que ser feito pela combinação da análise de regressão transversal com a análise de séries temporais, esta última do tipo longitudinal. Gujarati (1995) afirma que esse tipo de análise pode ajudar o modelo a dimensionar as repercussões das mudanças de atitudes e de padrões de comportamento ao longo do tempo. Adaptando-se (5) a esses pressupostos e considerando-se que $\ln A=\alpha_{0}, \Delta \ln Y_{i}=\Delta y_{i}$, $\Delta \ln L_{i}=\Delta l_{i}, \Delta \ln K_{1 i}=\Delta k_{1 i}, \Delta \ln K_{2 i}=\Delta k_{2 i}$ e $\Delta \ln K_{3 i}=\Delta k_{3 i}$, tem-se a seguinte formulação:

$$
\Delta y_{i t}=\alpha_{0}+\alpha_{1} \Delta l_{i t}+\beta_{1} \Delta k_{1 i t}+\beta_{2} \Delta k_{2 i t}+\beta_{3} \Delta k_{3 i t}+\varepsilon_{i t},
$$

sendo que $t$ é a referência ao ano analisado.

O estudo da influência exercida pela variabilidade dos elementos de TI na produção das empresas é uma questão fundamental para o alcance do objetivo deste trabalho, visto que, nos últimos sete anos, houve novos fatores que influenciaram o montante de investimentos em tecnologia da informação operado pelas empresas brasileiras. Do ponto de vista das empresas em geral, durante esse período houve uma exigência generalizada do incremento das práticas de governança corporativa devido ao desdobramento de uma crise fiduciária global ocasionada pelos escândalos de fraudes contábeis de mega-corporações americanas e européias. No Brasil, essa onda internacional tem ocasionado maior demanda por investimentos em tecnologia da informação, porquanto os órgãos de controle e o mercado de capitais têm exigido que as empresas nacionais cumpram com novos padrões de transparência da gestão. Do ponto de vista do setor financeiro, as novas medidas de minimização do risco do setor financeiro internacional estabelecidas a partir dos Acordos de Basiléia têm impulsionado os bancos a investir ainda mais em tecnologia da informação. 


\section{Fontes de Dados e Procedimentos Metodológicos da Análise de Dados em Painel}

Uma das razões da pouca abordagem brasileira do tema, tratado neste trabalho, diz respeito à escassez de dados para análise. De acordo com a especificação do modelo teórico e econométrico apresentado, percebe-se que há necessidade de informações que são de pouca divulgação e circulação, especialmente aquelas relativas ao valor dos ITI, do pessoal alocado em TI e do estoque físico de hardware. Dadas essas limitações da disponibilidade das informações, a especificação da equação (6) considerou somente o capital especificamente associado à tecnologia da informação, embora alguns dos estudos referenciados neste trabalho tenham incorporado uma variável representativa do capital não-associado especificamente à TI, para fazer um equilíbrio da função Cobb-Douglas.

No Brasil, a fonte pública e de maior confiabiliade de dados específicos de TI é a publicação anual da Revista INFO, que apresenta uma classificação das Cem Companhias mais Ligadas, onde constam especialmente dados de faturamento, investimentos realizados em TI, investimentos programados para TI, número de computadores, periféricos e servidores, número de profissionais de TI, e outras informações específicas de gestão de TI. Portanto, este trabalho tomou por base as informações divulgadas nesse periódico especializado, que é mantido por uma editora de reconhecida respeitabilidade, que é a Editora Abril S/A. Durante a digitação dos dados foram identificadas algumas inconsistências, relativas à repetição de valores e a ausência de dados de algumas empresas classificadas. Nesses casos, foi feita uma busca das informações necessárias junto às empresas e aos sites da Comissão de Valores Mobiliários [CVM] e de outros periódicos especializados em economia e finanças.

Por fim, adaptando-se (6) à terminologia dos dados pesquisados, tem-se:

$\Delta p_{i t}=\alpha_{0}+\alpha_{1} \Delta l t i_{i t}+\beta_{1} \Delta i t i_{i t}+\beta_{2} \Delta p c_{i t}+\beta_{3} \Delta s e r_{i t}+\varepsilon_{i t}$,

onde $p$ é o nível de produção, representado pela receita operacional líquida, da empresa analisada $i$ no ano $t$; lti representa o pessoal alocado em TI na empresa $i$ no ano $t$; iti representa o primeiro fator de capital, relativo ao valor dos investimentos em tecnologia da informação operados pela empresa $i$ no ano $t ; p c$ representa o segundo fator de capital, que é o estoque de microcomputadores mantido pela empresa $i$ no ano $t$ e ser representa o terceiro fator de capital, que se refere ao estoque de servidores mantido pela empresa $i$ no ano $t$. A representatividade das informações obtidas na fonte bibliográfica anteriormente descrita pode ser analisada por meio dos dados agregados na Tabela 1.

Tabela 1: Dados Agregados de Amostras de Empresas Líderes em Tecnologia da Informação no Brasil no Período de 2000 a 2006

\begin{tabular}{rrrrrrr}
\hline Ano & $\begin{array}{c}\text { Receita } \\
\text { (milhões US\$) }\end{array}$ & $\begin{array}{c}\text { Investimento TI } \\
\text { (milhões US\$) }\end{array}$ & $\begin{array}{c}\text { Profissionais } \\
\text { em TI }\end{array}$ & $\begin{array}{c}\text { Número de } \\
\text { PCs }\end{array}$ & $\begin{array}{c}\text { Número de } \\
\text { Servidores }\end{array}$ & $\begin{array}{c}\text { Amostra } \\
\text { Empresas }\end{array}$ \\
\hline 2000 & 83.270 & 1.436 & 16.182 & 344.493 & 15.207 & 96 \\
2001 & 100.624 & 2.040 & 28.244 & 645.414 & 41.991 & 99 \\
2002 & 97.944 & 1.584 & 20.083 & 629.264 & 43.418 & 100 \\
2003 & 143.614 & 2.291 & 28.344 & 854.020 & 64.376 & 100 \\
2004 & 187.272 & 2.880 & 29.716 & 822.254 & 53.192 & 98 \\
2005 & 194.533 & 2.286 & 18.436 & 709.659 & 52.064 & 96 \\
2006 & 253.044 & 2.621 & 21.315 & 820.960 & 56.203 & 97 \\
\hline
\end{tabular}

Fonte: adaptado de Editora Abril (2001, 2002, 2003, 2004, 2005, 2006, 2007).

De acordo com a Tabela 1, tem-se que a amostra anual de empresas apresenta números inferiores ao número de empresas que consta no ranking original. Isso se dá pelo fato de que algumas empresas foram excluídas das amostras anuais por não apresentarem todas as informações necessárias, mesmo após terem sido feitas buscas adicionais, como antes relatado. 
Embora as amostras anuais contenham vasto volume de informações, foram adotados procedimentos de adequação, a fim de que sua estrutura permita a análise de dados em painel. Como na análise de dados em painel se busca captar o comportamento de um determinado elemento dentro de um grupo de elementos ao longo do tempo, foi necessário redimensionar as amostras anuais. No processo de amostragem, que foi do tipo intencional, foram selecionadas as empresas que apresentaram maior constância nas classificações anuais e excluídas aquelas empresas que não figuraram em pelo menos três dos rankings anuais do período analisado. Na Tabela 2 tem-se uma síntese dos dados do redimensionamento que resultou em 429 observações válidas para um total de 98 empresas.

Tabela 2: Amostras Válidas de Empresas Líderes em Tecnologia da Informação no Brasil no Período de 2000 a 2006 e Setores de Atuação

\begin{tabular}{|c|c|c|c|c|c|c|c|c|c|c|}
\hline Ano & $\begin{array}{c}\text { Receita } \\
\text { (milhões US\$) }\end{array}$ & $\begin{array}{c}\text { Investimento TI } \\
\text { (milhões US\$) }\end{array}$ & $\begin{array}{c}\text { Profissionais } \\
\text { em TI }\end{array}$ & $\begin{array}{c}\text { Número de } \\
\text { PCs }\end{array}$ & $\begin{array}{l}\text { Número de } \\
\text { Servidores }\end{array}$ & $\begin{array}{c}\text { Amostra } \\
\text { Empresas }\end{array}$ & $\begin{array}{c}\text { Setor } \\
\text { Financeiro }\end{array}$ & $\begin{array}{c}\text { Setor } \\
\text { TI }\end{array}$ & $\begin{array}{c}\text { Setor } \\
\text { Serviços }\end{array}$ & $\begin{array}{c}\text { Setor } \\
\text { Industrial }\end{array}$ \\
\hline 2001 & 81.143 & 1.633 & 17.115 & 524.425 & 35.283 & 57 & 10 & 12 & 10 & 25 \\
\hline 2003 & 119.904 & 1.925 & 22.085 & 630.494 & 48.038 & 73 & 16 & 17 & 12 & 28 \\
\hline 2004 & 133.954 & 2.369 & 22.769 & 672.450 & 44.702 & 65 & 12 & 16 & 11 & 26 \\
\hline 2005 & 184.389 & 2.151 & 16.476 & 665.686 & 49.913 & 69 & 11 & 18 & 14 & 26 \\
\hline 2006 & 212.041 & 2.360 & 17.289 & 751.553 & 51.941 & 59 & 11 & 14 & 10 & 24 \\
\hline
\end{tabular}

Fonte: elaboração dos autores.

Como os dados válidos perfazem um total de 98 empresas (i), cujas amostras anuais variam no intervalo entre 73 e 44 empresas, tem-se uma situação em que os dados não estão balanceados, isto é, a unidade temporal $t$ não é constante para todos os elementos $i$. Enquanto os coeficientes angulares são comuns a todos os indivíduos, a estimação do intercepto $\alpha_{0}$ é um ponto crítico, quando se trata de um painel não-balanceado, que deve considerar o escopo do modelo econométrico na definição dos procedimentos de estimação a serem adotados, pois o intercepto pode capturar três tipos de efeitos: mistos, fixos e aleatórios; esses efeitos são originados pelos próprios grupos ou específicos ao tempo.

Neste trabalho, considerou-se que somente os efeitos dos grupos de indivíduos são relevantes e que os efeitos específicos ao tempo podem ser ignorados, de acordo com a argumentação técnica feita por Biørn (2004). Assim, foram estimados dois modelos, um deles incluindo a variável de estratificação de grupo e o outro não a considerando na análise. Com relação ao intercepto, partiu-se do pressuposto de que ele deve variar para cada indivíduo $i$, a fim de captar sua heterogeneidade não-observada, que é a característica do modelo de efeitos fixos.

De acordo com esses pressupostos, a estimação da equação (7) foi operacionalizada pelo software EA/LIMDEP (2002), de acordo com procedimentos descritos em Greene (2002), cujos resultados constam da Tabela 3.

Tabela 3: Resultados da Regressão da Produção como Função das Variáveis de TI

\begin{tabular}{|c|c|c|c|c|c|c|c|c|}
\hline \multirow[b]{2}{*}{ Variável } & \multicolumn{4}{|c|}{ Modelo 1 - Sem Variável Dummy de Grupo } & \multicolumn{4}{|c|}{ Modelo 2 - Com Variável Dummy de Grupo } \\
\hline & Coef. & St. Error & t-ratio & $\mathbf{P}|\mathbf{T}|>\mathbf{t}$ & Coef. & St. Error & t-ratio & $\mathbf{P}|\mathbf{T}|>\mathbf{t}$ \\
\hline Constante & $7,3111 * * *$ & 0,3595 & 20,3360 & 0,0000 & $8,2552 * * *$ & 0,4275 & 19,3090 & 0,0000 \\
\hline Número de funcionários em TI (lti ) & $-0,2482 * * *$ & 0,0529 & $-4,6940$ & 0,0000 & $-0,1234 * * *$ & 0,0458 & $-2,6910$ & 0,0071 \\
\hline Número de microcomputadores $(p c)$ & $0,7348 * * *$ & 0,0868 & 8,4690 & 0,0000 & $0,5144 * * *$ & 0,0860 & 5,9850 & 0,0000 \\
\hline Número de servidores (ser) & $-0,0355$ & 0,0752 & $-0,4730$ & 0,6366 & 0,0837 & 0,0686 & 1,2200 & 0,2226 \\
\hline
\end{tabular}




\section{Análise Empírica à Luz do Paradoxo da Produtividade}

Os resultados da Tabela 3 mostram que, nos dois modelos econométricos estimados, (H1) o nível de produção $(p)$ é positivamente sensível à mudança no nível de investimentos em TI (iti), ceteris paribus, o que refuta o Paradoxo da Produtividade. A refutação da hipótese do Paradoxo da Produtividade vai ao encontro dos resultados alcançados por estudos realizados no âmbito da OCDE (Pilat, 2004) e por estudiosos como Brynjolfsson (1993), Brynjolfsson e Hitt (1996), Osei-Bryson e Ko (2004) e Quan e Hu (2005). Além disso, os dois modelos apresentaram uma relação funcional estatisticamente significante entre a variável explicada e os insumos de tecnologia da informação número de funcionários em TI (lti) e estoque de microcomputadores $(p c)$ - o que torna a equação Cobb-Douglas balanceada para a posterior análise da eficiência econômica dos investimentos em tecnologia da informação.

Dentre os dois modelos estimados, o modelo que incorporou uma variável estratificada de grupo teve melhor aderência à especificação, especialmente por ter apresentado um coeficiente de correlação ajustado de $84,44 \%$, contra o coeficiente de $52,63 \%$ obtido pelo modelo 1 , o que comprova que o pressuposto da relevância dos efeitos dos grupos na análise foi alcançado. Além disso, o modelo 2 apresentou um valor $\mathrm{F}$ de 23,45 (significante a $1 \%$ ), o que atesta a validade de sua especificação. A fim de se testar a existência de efeitos fixos no intercepto, foi realizado o teste de Hausman, cujo resultado de 12,20 favorece a existência de efeitos fixos no intercepto, confirmando a premissa de que o intercepto variou para cada indivíduo $i$, tendo captado sua heterogeneidade não-observada.

\section{Análise de Eficiência Econômica dos Investimentos em Tecnologia da Informação}

Para a interpretação econômica da função Cobb-Douglas foi utilizada uma forma reduzida da equação (7):

$$
\Delta p_{i t}=\alpha_{0}+\alpha_{1} \Delta l t i_{i t}+\beta_{1} \Delta i t i_{i t}+\beta_{2} \Delta p c_{i t}+\varepsilon_{i t}
$$

na qual foi excluída a variável ser, pois não apresentou significância estatística, e reproduzidos os processos de modelagem e de estimação anteriormente descritos, o que resultou nos dados da Tabela 4. Os novos parâmetros estimados apresentam pouca variabilidade em relação àqueles constantes da Tabela 3 e os testes estatísticos também apresentaram e referendaram as suposições anteriormente estabelecidas $\left(\mathrm{R}^{2}=0,8813 ; \mathrm{F}=23,69\right.$; Hausman $\left.=9,78\right)$, o que atesta a robustez da estimação proposta.

Tabela 4: Resultados da Regressão Reduzida da Produção como Função das Variáveis de TI

\begin{tabular}{|c|c|c|c|c|c|c|c|c|}
\hline & Modelo 1 - Sem V & Tariável $D u$ & ummy de & Grupo & Modelo 2 - Com & Variável $D u$ & ummy de & Grupo \\
\hline Variável & Coef. & St. Error & t-ratio & $\mathbf{P}|\mathbf{T}|>\mathbf{t}$ & Coef. & St. Error & t-ratio & $\mathbf{P}|\mathbf{T}|>\mathbf{t}$ \\
\hline Constante & $7,3871 * * *$ & 0,3090 & 23,9070 & 0,0000 & $8,0726 * * *$ & 0,4016 & 20,1000 & 0,0000 \\
\hline Número de funcionários em TI (lti ) & $-0,2495 * * *$ & 0,0517 & $-4,8220$ & 0,0000 & $-0,1132 * * *$ & 0,0456 & $-2,4850$ & 0,0130 \\
\hline Número de microcomputadores $(p c)$ & $0,7060 * * *$ & 0,0681 & 10,3620 & 0,0000 & $0,5796 * * *$ & 0,0650 & 8,9240 & 0,0000 \\
\hline
\end{tabular}

Adaptando-se os parâmetros e os resultados dos testes estatísticos do modelo com a variável dummy de grupo à equação (7'), tem-se a seguinte função log-linear:

$$
\Delta p=8,0726-0,1132 \Delta l t i+0,1535 \Delta i t i+0,5796 \Delta p c,
$$

$$
(20,10) \quad(-2,48) \quad(4,17) \quad(8,92)
$$

que pode ser rearranjada de acordo com a função Cobb-Douglas (3), como segue:

$$
Y=3.205,37 L^{-0,1132} K_{1}^{0,1535} K_{2}^{0,5796} .
$$


sendo o intercepto 3.205,37 o valor do antilogaritmo neperiano de $\alpha_{0}$ e representa a incorporação do progresso tecnológico e da produtividade total dos fatores não-observados no modelo. Como fatores não-observados, têm-se especialmente os ganhos provenientes da eficiência de gestão, o retorno dos demais investimentos, que não são específicos da tecnologia da informação, e os ajustes nos preços dos produtos e serviços da empresa.

O expoente de cada uma das variáveis explicativas $x$ é o seu coeficiente de elasticidade-produto $\left(\varepsilon_{p / x}\right)$ estimado, pois mede seu impacto relativo no produto $p$. A análise agregada das elasticidades aponta a ocorrência de rendimentos decrescentes de escala, visto que seu somatório é menor que a unidade $(0,6199)$ e, nessa situação, o acréscimo nos insumos acarreta um acréscimo proporcionalmente menor no produto.

Portanto, embora o Paradoxo da Produtividade tenha sido refutado, a contribuição agregada dos insumos de tecnologia da informação no acréscimo na receita das empresas pesquisadas já encontrou seu ponto de saturação, confirmando a hipótese $\mathrm{H} 2$, sendo esta a mesma conclusão a que chegaram Lin e Shao (2006) e Dewan e Min (1997).

\section{Figura 1: Projeção da Curva de Rendimentos Decrescentes da Função de Produção Estimada}

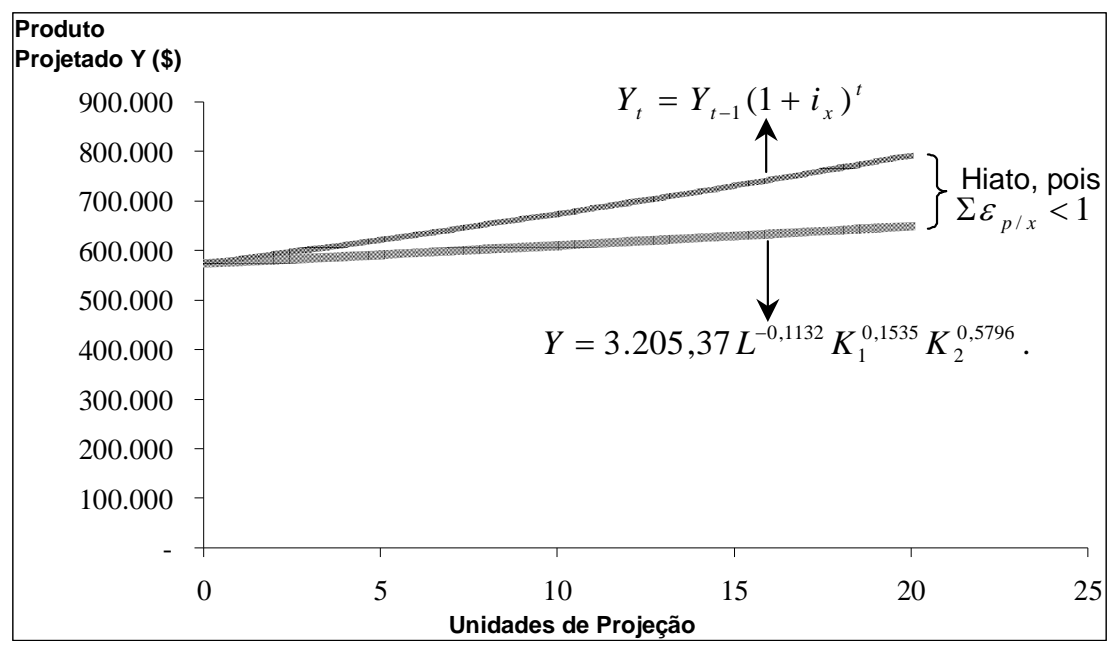

Fonte: elaboração dos autores.

A Figura 1 mostra a projeção do valor do produto $\mathrm{Y}$ em duas situações, tomando-se por base os valores médios dos insumos da amostra de empresas pesquisadas e considerando-se uma taxa de crescimento de $1 \%$ por período, com projeção para 20 períodos. Na primeira situação, tem-se a curva resultante da projeção do produto pela função Cobb-Doublas (8'), enquanto na segunda se tem a curva resultante da projeção do produto que considera sua expansão à taxa de crescimento geométrico dos insumos $\left(Y_{t}=Y_{t-1}\left(1+i_{x}\right)^{t}\right)$. A análise das duas curvas traçadas evidencia a ocorrência de rendimentos de escala, mostrando que o hiato é crescente a uma taxa geométrica, pelo fato de que o acréscimo de $1 \%$ nos insumos ocasiona um acréscimo proporcionalmente menor no produto, de cerca de $0,619 \%$.

Enquanto a análise agregada apontou o desempenho do conjunto dos insumos, a análise do comportamento de cada insumo possibilita a identificação dos pontos que necessitam de atenção, tomando por base a análise individual das elasticidades num horizonte temporal de curto prazo, para o qual se aplica a condição ceteris paribus. Essa análise individual tomou por base os mesmos procedimentos metodológicos da projeção anteriormente relatada e incluiu, além do produto projetado com a variação conjunta dos insumos $(\mathrm{p}(\mathrm{E}=0,619))$, o produto projetado para cada um dos insumos isoladamente, considerando-se a condição ceteris paribus. 


\section{Figura 2: Projeção do Produto para Análise Agregada e Individual dos Insumos}

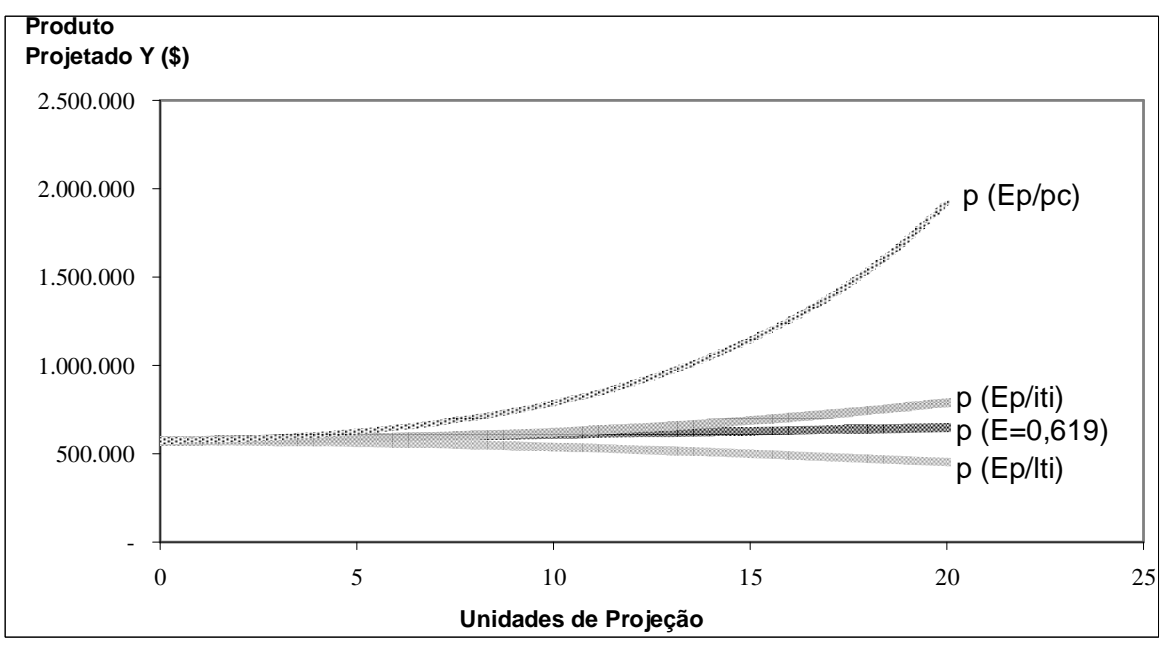

Fonte: elaboração dos autores.

Dentre as curvas traçadas na Figura 2, verifica-se que aquela pertencente ao insumo pessoal alocado em TI é correlacionada com uma redução na variação do produto $p$, pois tem uma elasticidade-produto $\left(\varepsilon_{p / t i}\right)$ de -0.1132 , quando os demais insumos permanecerem constantes. Essa relação funcional requer atenção, pois ela é a grande responsável pelos rendimentos de escala decrescentes obtidos nesta modelagem. Adicionalmente, os investimentos em tecnologia da informação são correlacionados com um acréscimo estatisticamente significante de aproximadamente $0,1535\left(\varepsilon_{p / i t i}\right)$ no produto, caso os demais insumos estejam constantes; a elasticidade-produto do estoque físico de computadores $\left(\varepsilon_{p / p c}\right)$ apresentou uma relação estatisticamente significante, com um impacto de cerca de 0,5796 no acréscimo do produto, ceteris paribus.

Uma análise mais apurada do impacto dos investimentos em tecnologia da informação, dado que a justificativa econômica desse item é fundamental no processo decisório da análise de investimentos, conforme postulado na hipótese $\mathrm{H} 3$, pode ser feita a partir do conceito de produtividade marginal do insumo, que indica o produto adicional que poderia ser esperado pelo acréscimo de uma unidade do insumo $x\left(P M_{x}\right)$, ceteris paribus.

Para o caso da produtividade marginal dos investimentos em tecnologia da informação, tem-se a seguinte dedução, que é baseada na proposta de Pereira (2000) e que, em parte, também foi aplicada por Brynjolfsson e Hitt (1996):

$\varepsilon_{p / i t i}=\frac{\Delta \ln p}{\Delta \ln i t i} ; P M_{i t i}=\frac{\Delta p}{\Delta i t i} ; P M_{i t i}=\varepsilon_{p / i t i} \cdot \frac{p}{i t i}$.

A equação (9) mostra que a produtividade marginal dos investimentos em tecnologia da informação representa sua taxa de retorno acumulada no período da análise, como uma função do coeficiente de elasticidade do produto $p\left(\varepsilon_{p / i t i}\right)$ multiplicado pela razão produto-investimento em tecnologia da informação ( $p /$ iti $)$, considerando-se seus valores agregados.

Para o cálculo da taxa interna de retorno $\left[\mathrm{TIR}_{i}\right]$ anual da empresa $i$, toma-se sua taxa de retorno acumulada $\left(\mathrm{MP}_{i}\right)$, que deve ser descapitalizada pelo seu número de observações $n$, conforme a equação: $\operatorname{TIR}_{i}=\left[\left(1+P M_{i}\right)^{1 / n}-1\right] \times 100$, e $n$ varia para cada empresa, pois se trata de um painel nãobalanceado de dados. Logo, o valor final da taxa interna de retorno é uma medida de rentabilidade média anual bruta dos investimentos. Na Tabela 5, têm-se os resultados dos cálculos do produto marginal e da taxa interna de retorno, em termos globais (IG) e em termos setoriais. 
Tabela 5: Taxa Interna de Retorno Anual Bruta dos Investimentos em Tecnologia da Informação

\begin{tabular}{lccccc}
\hline & Empresas & TIR $_{\boldsymbol{i}}$ & Desvio-padrão & TIR $_{\boldsymbol{i}}$ Máx & TIR $_{\boldsymbol{i}}$ Mín \\
\hline Índice Global & 98 & $125.28 \%$ & $86.04 \%$ & $448.99 \%$ & $16.56 \%$ \\
Financeiro & 18 & $85.93 \%$ & $73.74 \%$ & $292.91 \%$ & $18.10 \%$ \\
Tecnologia da Informação & 24 & $103.99 \%$ & $55.62 \%$ & $232.80 \%$ & $16.56 \%$ \\
Serviços & 16 & $164.07 \%$ & $102.80 \%$ & $448.99 \%$ & $57.98 \%$ \\
Industrial & 40 & $164.44 \%$ & $102.04 \%$ & $400.08 \%$ & $51.79 \%$ \\
\hline
\end{tabular}

$\mathrm{E}_{p / i t i}=0,1535$

Fonte: elaboração dos autores.

A série geral de taxas internas de retorno brutas tem uma distribuição normal, com uma média anual de $125.28 \%$ para o período de 2000 a 2006 e um desvio-padrão de $86.04 \%$, como pode ser visto na Figura 3, cujos gráficos foram operacionalizados pelo software Minitab15 30day Trial. Nessas condições, e tomando-se como custo de oportunidade do capital [COP] a taxa média anual bruta SELIC de $18.20 \%$ no período analisado, pode-se rejeitar a hipótese $\mathrm{H} 3$, pois há eficiência marginal do capital investido em tecnologia da informação, visto que o retorno médio encontrado supera seu custo de oportunidade $(T I R=125.28 \%>C O P=18.20 \%)$. Essa taxa de $125.28 \%$ ao ano é cerca de $44 \%$ superior aos $81 \%$ encontrados no estudo publicado há mais de uma década por Brynjolfsson e Hitt (1996). Uma possível explicação para essa discrepância, partindo-se do pressuposto de que ela não encontra fundamentos na variação monetária ocorrida no período entre os dois estudos, pode estar associada ao âmbito regional da pesquisa. Nesse sentido, e considerando-se que as taxas de retorno são mais altas, quando esse tipo de investimento ocorre sobre uma base de uso menos intensivo de tecnologia da informação, conforme constatado por Dewan e Kraemer (2000), pode-se supor que a taxa de retorno dos ITI dos países em desenvolvimento é mais alta do que em países desenvolvidos, o que justificaria a diferença acima citada entre os resultados.

Os gráficos expostos na Figura 3 permitem algumas interpretações no âmbito setorial. O setor financeiro tem a mais baixa taxa de retorno média, com $85.93 \%$ ao ano e, à medida que aumentam os valores dos investimentos em tecnologia da informação, tem-se uma redução em sua taxa de retorno. Uma explicação para esse comportamento pode estar relacionada ao fato de que o setor financeiro é o setor que usa mais intensivamente a tecnologia da informação, conforme apontam os estudos feitos pela OCDE (Pilat, 2004). E, considerando-se que o setor financeiro brasileiro tem acompanhado o mesmo nível de utilização de fatores de tecnologia da informação adotado pelos líderes no sistema financeiro internacional, suas taxas de retorno são diretamente afetadas pela maior exposição aos custos complementares que o uso de tecnologias mais avançadas costumam apresentar (Atzeni \& Carboni, 2006).

No caso dos demais setores pesquisados, o setor que apresentou a maior taxa média de retorno anual foi o setor industrial, com $164.4 \%$, que é seguido pelo setor de serviços com $164.1 \%$ e, por fim, tem-se o setor de tecnologia da informação com 104\%. Especialmente no setor industrial e no setor de serviços, as taxas de retorno costumam permanecer na média setorial, à medida que há aumento nos investimentos em TI. Ao se considerar que esses setores são aqueles que utilizam menor quantidade de fatores de TI, pode-se reforçar a suposição de que há maior eficiência nos investimentos aplicados em empresas de uso menos intensivo de tecnologia da informação. 
Figura 3: Distribuição do Retorno dos Investimentos em Tecnologia da Informação nos Setores

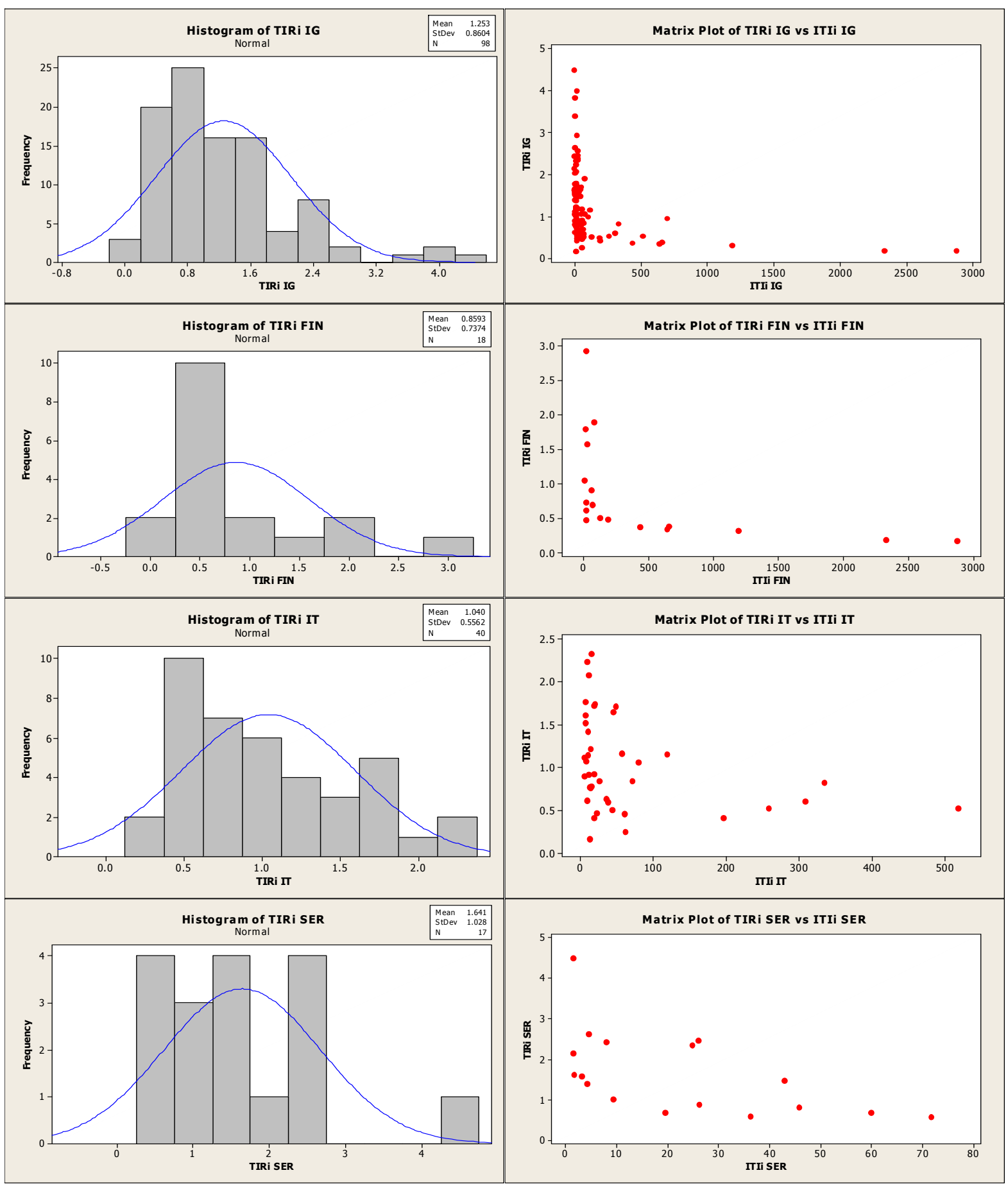




\section{(conclusão)}

Figura 3: Distribuição do Retorno dos Investimentos em Tecnologia da Informação nos Setores

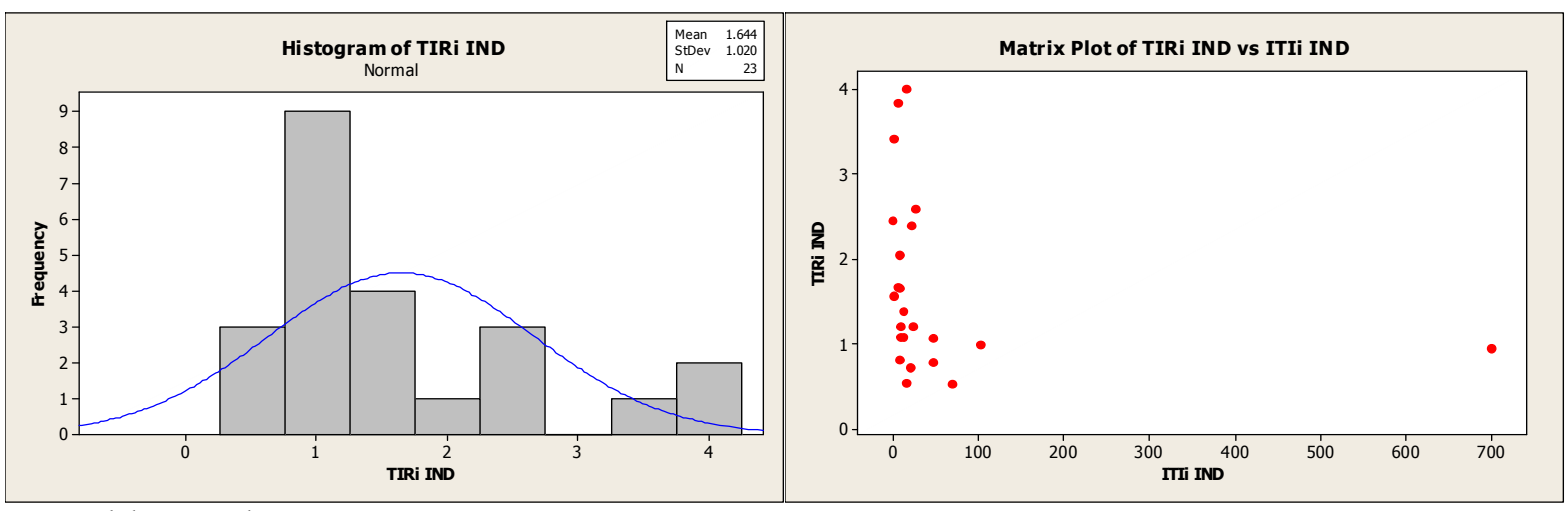

Fonte: elaboração dos autores.

\section{CONSIDERAÇõES FINAIS E RECOMENDAÇÕES}

O Paradoxo da Produtividade é tema controverso e polêmico, que oferece ampla gama de estudos, a maioria dos quais baseados em diferentes pressupostos, sistemas de hipóteses e com metodologias próprias para modelagem, que têm produzido resultados que ora convergem para sua refutação e ora convergem para sua confirmação. Há estudos cujos testes de hipóteses tomaram o conjunto de fatores de tecnologia da informação, enquanto outros consideraram somente os investimentos em tecnologia da informação.

Neste trabalho, adotou-se a segunda corrente de estudos na definição da hipótese central, pois o teste do Paradoxo da Produtividade foi realizado à luz dos investimentos em tecnologia da informação. No entanto, a fim de tornar o alcance deste estudo mais amplo, também foi considerada no sistema de hipóteses a análise da eficiência econômica do conjunto de fatores de tecnologia da informação na produtividade das empresas analisadas.

O Paradoxo da Produtividade foi refutado na amostra de empresas pesquisadas, pois foi identificada uma relação significante e positiva entre o acréscimo dos investimentos em tecnologia da informação e o acréscimo na produção das empresas analisadas, ceteris paribus. A amostra pesquisada foi constituída de 429 observações de 98 empresas, que faturaram no período entre os 2000 e 2006 um valor aproximado de US\$ 856 bilhões e operaram investimentos em tecnologia da informação da ordem de US\$12,9 bilhões.

Em termos de eficiência agregada dos fatores de tecnologia da informação, foram identificados indícios de saturação na relação funcional modelada. A análise agregada apontou a presença de rendimentos decrescentes de escala, comprovando a segunda hipótese deste trabalho. Esse comportamento é motivado especialmente pela variável número de funcionários em tecnologia da informação, que apresentou um coeficiente de elasticidade de -0.1132 . Como neste trabalho se tomou como descritor da produção empresarial o volume de vendas, e considerando-se que esse volume é um indicador do porte da organização, pode-se inferir que o aumento na escala de produção tem sido acompanhado de reduções na eficiência econômica do trabalho empregado em tecnologia da informação.

Em termos de análise individual, as demais variáveis, investimentos em tecnologia da informação e estoque físico de computadores, têm tido um impacto positivo na variação da receita, o que atesta sua eficiência no desempenho das organizações pesquisadas. Uma análise mais profunda da produtividade marginal dos investimentos em tecnologia da informação e de sua derivação na taxa interna de retorno anual concluiu pela rejeição da terceira hipótese do trabalho. A eficiência marginal do capital foi 
comprovada pelas taxas brutas de retorno apresentadas pelos investimentos em TI que, na média, se situaram em $125.5 \%$ ao ano e são superiores ao custo médio de oportunidade do capital do período $(18.20 \%)$.

Não obstante os resultados do trabalho mostrem que, em geral, os maiores retornos dos investimentos em tecnologia da informação têm ocorrido nas maiores escalas de produção, verificouse que o setor financeiro apresentou comportamento distinto daquele dos demais setores pesquisados. O setor financeiro apresentou a menor taxa de retorno médio (85.93\% ao ano), que é tecnicamente a mesma taxa apresentada pelo estudo de Brynjolfsson e Hitt (1996). Esse fato pode explicar que os retornos são menores, quando operados sobre uma base de uso mais intensivo de fatores de tecnologia da informação, que é algo intrínseco ao setor financeiro, mesmo no Brasil. Os demais setores de uso menos intensivo de tecnologia da informação, como o setor industrial e de serviços, apresentaram as maiores taxas brutas médias de retorno, com $164.4 \%$ e $164.1 \%$, respectivamente, enquanto o setor de tecnologia da informação apresentou uma taxa bruta média de retorno de $104 \%$.

Embora esses resultados devam ser vistos com reservas, em função das limitações usuais das fontes de dados, do processo de modelagem e da análise econométrica, concluiu-se que eles podem dar suporte ao processo decisório do planejamento de investimentos e de estratégias de negócios em tecnologia da informação. Para as empresas que exploram a tecnologia da informação como a principal atividade do negócio, a posse de informações sobre a distribuição de taxas de retorno por setor da atividade econômica poderia possibilitar o direcionamento de suas estratégias de negócios àqueles setores que apresentaram maiores possibilidades de retorno. Da mesma forma, tais empresas poderiam planejar estratégias de negócios alternativos com vistas à reversão do problema do suposto ponto de saturação do acréscimo de mão-de-obra específica à TI, por exemplo, por meio do reforço de políticas de terceirização.

Do ponto de vista das empresas que operam investimentos em tecnologia da informação, onde a TI não representa a atividade principal do negócio, a disponibilidade de taxas de retorno setoriais, com seus respectivos desvios-padrão, possibilitaria que esses dados sejam considerados como parâmetros relativos para efeito de comparação na aplicação das técnicas de fluxo de caixa descontado. Assim, após uma empresa calcular a taxa interna de retorno de seus investimentos em tecnologia da informação, ela poderia analisar seu desempenho em comparação com as demais empresas de seu setor, a partir de seu posicionamento na faixa da escala de rentabilidade setorial.

Por fim, tem-se que este trabalho, além de ter evidenciado a refutação do Paradoxo da Produtividade, tomando por base um sistema de hipóteses robusto, apresentou como contribuições os procedimentos metodológicos para a análise de dados em painel não-balanceados oriundos de fontes especializadas e os procedimentos de modelagem da função Cobb-Douglas para o cálculo da eficiência econômica dos insumos, que podem dar suporte ao processo decisório de análise de investimentos em tecnologia da informação. Esta pesquisa pode ter novos desenvolvimentos, entre os quais a inclusão de outros fatores de produção que não sejam específicos à tecnologia da informação no processo de modelagem.

Artigo recebido em 28.02.2008. Aprovado em 20.08.2008.

\section{REFERENCIAS BIBLIOGRÁFICAS}

Atzeni, G. E., \& Carboni, O. A. (2006). ICT productivity and firm propensity to innovative investment: evidence from Italian microdata. Information Economics and Policy, 18(2), 139156.

Banker, R. D., \& Kauffman, R. J. (2004). The evolution of research on information systems: a fiftiethyear survey of the literature. Management Science, 50(3), 281-298. 
Baumol, W. J. (1977). Economic theory and operations analysis. London: Prentice-Hall.

Bharadwaj, A. S., Bharadwaj, S. G., \& Konsynski, B. R. (1999). Information technology effects on firm performance as measured by Tobin’s. Management Science, 45(6), 1008-1024.

Biørn, E. (2004). Regression system for unbalanced panel data: a stepwise maximum likelihood procedure. Journal of Econometrics, 122(2), 281-291.

Brynjolfsson, E. (1993). The productivity paradox of information technology. Communications of the ACM, 36(12), 66-77.

Brynjolfsson, E., \& Hitt, L. M. (1995). Information technology as a factor of production: The role of differences among firms. Economics of Innovation and New Technology, 3(3-4), 183-199.

Brynjolfsson, E., \& Hitt, L. (1996). Paradox lost? Firm-level evidence on the returns to information systems spending. Management Science, 42(4), 541-558.

Brynjolfsson, E., \& Hitt, L. M. (1998). Beyond the productivity paradox. Communications of the ACM, 41(8), 49-55.

Brynjolfsson, E., \& Hitt, L. M. (2000). Beyond computation: information technology, organizational transformation and business performance. Journal of Economic Perspectives, 14(4), 23-48.

Brynjolfsson, E., \& Yang, S. (1996). Information technology and productivity: a review of the literature. Advances in Computers, 43(1), 179-214.

Chiang, A. C. (1974). Fundamental methods of mathematical economics. Tokyo: McGraw-Hill Kogakusha.

Cobb, C. W., \& Douglas, P. H. (1928). A theory of production. American Economic Review, 18(1), 139-165.

Dewan, S., \& Kraemer, K. L. (2000). Information technology and productivity: evidence from country-level data. Management Science, 46(4), 548-562.

Dewan, S., \& Min, C. (1997). The substitution of information technology for other factors of production: a firm-level analysis. Management Science, 43(12), 1660-1675.

EA/LIMDEP. (2002). Econometric Analysis Software (Version 1.1.). Plainview, New York: Econometric Software.

Editora Abril (2001). Revista Infoexame: as 100 empresas mais ligadas do Brasil. São Paulo, SP: Autor.

Editora Abril (2002). Revista Infoexame: as 100 empresas mais ligadas do Brasil. São Paulo, SP: Autor.

Editora Abril (2003). Revista Infoexame: as 100 empresas mais ligadas do Brasil. São Paulo, SP: Autor.

Editora Abril (2004). Revista Infoexame: as 100 empresas mais ligadas do Brasil. São Paulo, SP: Autor.

Editora Abril (2005). Revista Infoexame: as 100 empresas mais ligadas do Brasil. São Paulo, SP: Autor.

Editora Abril (2006). Revista Infoexame: as 100 empresas mais ligadas do Brasil. São Paulo, SP: Autor. 
Editora Abril (2007). Revista Infoexame: as 100 empresas mais ligadas do Brasil. São Paulo, SP: Autor.

Engelbrecht, H. J., \& Xayavong, V. (2006). ICT intensity and New Zealand's productivity malaise: is the glass half empty or half full? Information Economics and Policy, 18(1), 24-42.

Greene, W. H. (2002). LIMDEP Version 8.0 econometric modeling guide (4th ed.). Plainview, New York: Econometric Software.

Gujarati, D. N. (1995). Basic econometrics. New York: Mc-Graw-Hill, Inc.

Gurbaxani, V., Melville, N., \& Kraemer, K. (1998). Disaggregating the return on investment to IT capital. Center for research on information technology and organizations. Berkeley: University of California.

Gurbaxani, V., Melville, N., \& Kraemer, K. (2000). The production of information services: a firmlevel analysis of information systems budgets. Information Systems Research, 11(2), 159-176.

Humphrey, T. M. (1997). Algebraic production functions and their uses before Cobb-Douglas. Economic Quarterly - Federal Reserve Bank of Richmond, 83(1), 51-83.

Lin, W. T., \& Shao, B. B. M. (2006). The business value of information technology and inputs substitution: the productivity paradox revisited. Decision Support Systems, 42(2), 493-507.

Menezes, R. B., \& Moura, H. J. (2004, setembro). Revisitando o paradoxo da produtividade de tecnologia da informação (TI): estudo de caso de um banco brasileiro. Anais do Encontro Nacional da Associação Nacional de Pós-Graduação e Pesquisa em Administração, Curitiba, PR, Brasil, 28.

Milana, C., \& Zeli, A. (2002). The Contribution of ICT to production efficiency in Italy: firm-level evidence using data envelopment analysis and econometric estimations [Working Paper $\mathrm{N}^{\circ}$ 2002/13STI]. Organisation for Economic Co-operation and Development, Paris, France.

O'mahony, M., \& Vecchi, M. (2005). Quantifying the impact of ICT capital on output growth: a heterogeneous dynamic panel approach. Economatica, 72(288), 615-633.

Osei-Bryson, K. M., \& Ko, M. (2004). Exploring the relationship between information technology investments and firm performance using regression splines analysis. Information \& Management, 42(1), 1-13.

Pereira, A. M. (2000). Is all public capital created equal? Review of Economics and Statistics, 82(3), 513-518.

Peslak, A. R. (2003). A firm level study of information technology productivity by industry using financial and market based measure. Journal of Information Technology Impact, 3(2), 77-90.

Pilat, D. (2004). The ICT productivity paradox: insights from micro data. OECD Economic Studies, $38,37-65$.

Quan, J. J., \& Hu, Q. (2005). Evaluating the impact of IT investments on productivity: a causal analysis at industry level. International Journal of Information Management, 25(1), 39-53.

Sabyasachi, M. (2005). Information technology as an enabler of growth in firms: an empirical assessment. Journal of Management Systems, 22(2), 279-300.

Santos, T. (1987). Revolução científico-técnica e a acumulação do capital. Petrópolis: Vozes.

Solow, R. M. (1987, July 12). We'd better watch out. New York Times Book Review, 36. 
Solow, R. M. (1988). Growth theory and after. The American Economic Review, 78(3), 307-317.

Teixeira, F. L. C. (1998, setembro). O paradoxo de Solow e o debate sobre tecnologia e produtividade no Brasil. Anais do Encontro Nacional da Associação Nacional de Pós-Graduação e Pesquisa em Administração, Foz do Iguaçu, PR, Brasil, 22. 